\title{
Examining the Baby Café Model and Mothers' Breastfeeding Duration, Meeting of Goals, and Exclusivity
}

\author{
Lucia A. Jenkins, ${ }^{1}$ Katie Barnes, ${ }^{2}$ Angela Latter, ${ }^{3}$ and Roger A. Edwards ${ }^{4}$
}

\begin{abstract}
Objective: Accessible community lactation support impacts a woman's breastfeeding success by offering timely intervention and solutions, thereby allowing mothers to achieve breastfeeding goals and improve overall breastfeeding rates. Although the impact of breastfeeding support has been well established, there is a lack of consistency in the development and evaluation of support models. This report examines two differing populations of Baby Café attendees. The study evaluated the mother's achievement of personal and nationally recommended breastfeeding goals, the frequency of attending a Baby Café, and their ratings of the program as helpful in solving breastfeeding problems.

Methods: A total of 559 mothers attending two Baby Cafés, one in Massachusetts and the other in southern Texas, were surveyed when their babies were 6 months old and again over age 12 months. Actual breastfeeding duration was compared with the mothers' initially stated goals and American Academy of Pediatrics (AAP) recommended goals, and then evaluated against the number of Café attendances. The mother's rating of the Café for helpfulness was measured using a 1-5 effectiveness scale.

Results: Results show that mothers attending either of the surveyed Baby Cafés that served distinctly different populations reported higher breastfeeding exclusivity rates and higher rates of 12-month breastfeeding duration than national rates reported by the Centers for Disease Control and Prevention (CDC). More than 70\% of all mothers surveyed rated the Café as most effective.

Conclusions: The Baby Café model was shown to be effective at helping mothers reach breastfeeding goals regardless of the Café's different geographical settings and the socioeconomic characteristics of the populations served.
\end{abstract}

Keywords: breastfeeding, community support, duration, exclusivity, goals

\section{Introduction}

B ABy CAFÉ USA, Inc., (BCUSA), 501(c)(3), was established in 2011 to promote the development of licensed, drop-in, free-of-charge, and standardized breastfeeding support sites offering peer support coupled with professional lactation care. Licensing requires approval by BCUSA, and includes program operation materials, web presence, and training for all staff. Specific standards include regular weekly sessions offering comfortable informal seating, breastfeeding-positive messaging, snacks, a digital scale, and the presence of an International Board-Certified Lactation Consultant (IBCLC), or oversight by an IBCLC when the café is staffed with alternative approved breastfeeding counselors.
Many cafes also offer guest speakers on various helpful subjects, and Facebook ${ }^{\circledR}$ forums where mothers connect socially, sharing breastfeeding knowledge and experiences.

In efforts to increase awareness and accessibility to all mothers, Baby Cafés are required to establish connections with appropriate surrounding community programs such as Special Supplemental Nutrition Program for Women, Infants, and Children (WIC), Visiting Nurse Association (VNA), and Healthy Start, and have referral points for necessary medical or psychosocial intervention. Statistical data are collected by all cafes and submitted to BCUSA for analysis.

There are currently 1,544 licensed Cafés in 31 states, providing support to thousands of mothers, and increasing lactation access to underserved areas with low breastfeeding

\footnotetext{
${ }^{1}$ Baby Café USA, Inc., Wakefield, Massachusetts, USA.

${ }^{2}$ Community Education Department, Melrose-Wakefield Hospital (MWH), Melrose, Massachusetts, USA.

${ }^{3}$ Nursing Faculty, Worcester State University, Worcester, Massachusetts, USA.

${ }^{4}$ Center for Interprofessional Studies and Innovation, MGH Institute of Health Professions, Charlestown, Massachusetts, USA.
}

(C) Lucia A. Jenkins et al., 2020; Published by Mary Ann Liebert, Inc. This Open Access article is distributed under the terms of the Creative Commons Attribution Noncommercial License (http://creativecommons.org/licenses/by-nc/4.0/) which permits any noncommercial use, distribution, and reproduction in any medium, provided the original author(s) and the source are cited. 
rates. The average annual operation expense is $\$ 11,000$. Cafés are individually funded by sponsoring organizations using a range of national, community, and private grants, such as block and obesity grants. Baby Cafés are World Health Organization (WHO) Code compliant ${ }^{1}$ and fulfill Step 10 of the Baby-Friendly Hospital Initiative (BFHI) requirements. ${ }^{2}$

Currently, $83.2 \%$ of U.S. mothers initiate breastfeeding, but contrary to Centers for Disease Control and Prevention (CDC) recommendations, only $24.9 \%$ exclusively breastfeed for the recommended 6 months, with $35.9 \%$ continuing to 1 year. ${ }^{3}$ Healthy People 2020 (HP2020), the Surgeon General's Call to Action, and BFHI all identify a need for increased efforts by both health care providers and the community to help mothers reach breastfeeding goals by establishing accessible support systems. ${ }^{2,4,5}$ Current literature confirms that breastfeeding support from family, friends, and health care workers significantly impacts a woman's decision to breastfeed. The literature further identifies postpartum community support as crucial for a successful breastfeeding experience. ${ }^{6}$ Although multiple studies have determined the need for breastfeeding support groups, there is a lack of consistency in the development and evaluation of community models. There is limited research that has defined the combination IBCLC and peer support as one of the successful components of support groups. ${ }^{7,8}$ This report describes the Baby Café program, with its required standards and practices, that may provide a model for nationwide network of professional community support activities.

\section{Methods}

Breastfeeding mothers from two separate Baby Cafés, one in Massachusetts and the other in Texas, were chosen for this study. The Massachusetts Baby Café (Melrose-Wakefield Hospital $[\mathrm{MWH}]$ ) is 30 minutes outside Boston: attending mothers birthed in Boston and surrounding area hospitals. The Texas Baby Café (San Antonio [SA]) is sited in five SA urban WIC offices and is open to both WIC and non-WIC mothers. The MWH Café population is $83 \%$ Caucasian with $3 \%$ using WIC, whereas the SA population is $82 \%$ Hispanic with 99\% using WIC (Table 1). All surveyed mothers desired to breastfeed and self-selected to attend the Baby Cafés.

Baby Cafés collected three types of data: initial registration and demographics, weekly attendance and exclusivity, and outcome survey data. Institutional Review Board (IRB) approval was received from MWH. This report used initial registration, exclusivity, and outcome survey data for analysis.

At first attendance, individual mothers register with their first name, contact information, their baby's first initial and age, start date of any formula use, and personal breastfeeding duration goal. Each mother is assigned an ID number, protecting her privacy. Of the 563 mothers surveyed, 4 had "no goal" and were excluded from analysis, leaving a total of 559 mothers: 150 from MWH Café and 409 from SA Café.

A point-in-time survey was given to the mother at her baby's age of just over 6 months, and again over 12 months. ${ }^{9}$ Surveys were administered through phone call, e-mail, or in person at Baby Café. Mothers reported their current breastfeeding status, beginning and ending dates of formula use, the Café's usefulness, and whether they attended five times or more. The attendance parameter "five times or more" was based on the
Table 1. Demographics of Mothers Attending MWH AND SA BABY CAFÉS

\begin{tabular}{lrr}
\hline Mother's age & MWH & \multicolumn{1}{c}{ SA } \\
\hline Under 19 years & $0.00 \%$ & $12.05 \%$ \\
BTW 19 \& 25 & $5.26 \%$ & $46.58 \%$ \\
Over 25 years & $94.74 \%$ & $53.42 \%$ \\
Ethnicity & $6.49 \%$ & \\
Asian & $2.60 \%$ & $0.85 \%$ \\
Black American & $0.00 \%$ & $6.25 \%$ \\
Black other & $83.12 \%$ & $0.57 \%$ \\
Caucasian & $5.19 \%$ & $9.09 \%$ \\
Hispanic & $2.60 \%$ & $81.82 \%$ \\
Indian & $0.00 \%$ & $0.00 \%$ \\
Other & & $1.42 \%$ \\
Education & $1.33 \%$ & \\
High school or GED & $36.00 \%$ & $79.94 \%$ \\
College degree & $61.33 \%$ & $11.60 \%$ \\
Postgraduate degree & $1.33 \%$ & $5.02 \%$ \\
In school & & $3.45 \%$ \\
Miscellaneous & $2.63 \%$ & $99.69 \%$ \\
Using WIC & $73.68 \%$ & $19.94 \%$ \\
Working outside home & & \\
\hline
\end{tabular}

GED, General Education Diploma; MWH, Melrose-Wakefield Hospital; SA, San Antonio; WIC, Special Supplemental Nutrition Program for Women, Infants, and Children.

average number of American Academy of Pediatrics (AAP) recommended pediatric well visits in the first 6 months, when feeding difficulties are often discovered. ${ }^{10}$ If weaned at the time of the survey, the month of wean age was recorded.

Mothers rated the helpfulness of the Café on a scale of 1-5 ( 1 being not helpful, and 5 extremely helpful), and offered comments on the program. Wean age was compared with the mother's intended breastfeeding duration. The mother's success meeting her initial duration goal was compared with her Café attendance category of "five times or more" or "less than five times." Outcomes were defined as falling short of, meeting/and or exceeding own goal, and meeting AAP-recommended 12-month goal.

Formula usage was divided into age brackets closely aligned to the AAP standard visit time frames, specifically, birth -3 weeks; $3-6$ weeks; 6 weeks -3 months; 3-6 months; 6-12 months, and 1-3 years. Formula supplementation rates were calculated and resulting breastfeeding exclusivity rates were determined for each age group. Exclusivity is defined as baby receiving only breast milk within each time frame (up to 6 months) and receiving only breast milk and solids from 6 to 12 months. Exclusivity rates were compared with those published by the CDC. ${ }^{3}$ Chi-square analysis was used to compare Baby Café attendance with mothers' success meeting the AAP and personal breastfeeding duration goals. A publicly available chi-square calculator from Social Science Statistics website was used to determine whether the results were statistically significant.

\section{Results}

AAP goal: 326 (58\%) of the surveyed mothers met the AAP goal of breastfeeding for 12 months, significantly higher than the national CDC national rate of $35.9 \%$. Of the 559 total mothers, 127 (23\%) attended Baby Café "five times or 
Table 2. Comparison of Baby Café Attendance AND Mothers ACHIEVING THE AAP 12-Month

BreastFeEding Duration Goal, COMPARED WITH CDC NaTional 12-Month

BREASTFEEDING RATES

\begin{tabular}{lccc}
\hline & $\begin{array}{c}\text { Baby Café } \\
\text { BF@ 12 months }\end{array}$ & $\begin{array}{c}\text { National } \\
\text { CDC BF@ 12 } \\
\text { months rate }\end{array}$ \\
\cline { 2 - 4 } & & $n 326$ & $\mathrm{n} / \mathrm{a}$ \\
$\begin{array}{l}\text { Number of visits } \\
\begin{array}{c}\text { Met AAP goal } \\
\text { 12 months }\end{array}\end{array}$ & $30 \%$ & $70 \%(p=0.0023)$ & $35.9 \%$ \\
\hline
\end{tabular}

AAP, American Academy of Pediatrics; CDC, Centers for Disease Control and Prevention.

more." Of the high attendance mothers, 89 (70\%) met the AAP breastfeeding goal. The chi-square analysis of mothers meeting the 12-month goal resulted in a statistically significant difference $(p<0.05)$ between those who attended "less than five times" and those who attended "five times or more," $\chi^{2}=9.35$ (1), $p=0.0023$ (Table 2).

Personal goal: $77 \%(\mathrm{MWH})$ and $79 \%(\mathrm{SA})$ of surveyed mothers met and/or exceeded their personal breastfeeding duration goals and were more likely to have attended the Cafés "five times or more." There was no statistically significant difference between meeting personal goals and number of attendances (Table 3).

Exclusivity: The CDC reports national breastfeeding exclusivity rates of $47 \%$ at 3 months and $25 \%$ at 6 months. The MWH mothers reported exclusivity rates of $77 \%$ at 3 months and $71 \%$ at 6 months. These rates are 1.64 and 2.84 times higher at 3 and 6 months, respectively, than the rates of the total breastfeeding population published by CDC. Mothers in SA reported similar rates (Table 4).

Mothers' ratings of both Café's helpfulness were high, with the percentages as follows: extremely helpful $75.37 \%$; very helpful $16.30 \%$; helpful $6.30 \%$; slightly helpful $2.04 \%$; and not helpful $0.00 \%$. The comments were very positive for the combination of professional/peer support, with frequent mentions of "I learned so much"; "Café solved my issue when I was going to quit and I'm still breastfeeding"; "Great breastfeeding experts with the answers," and "I wouldn't be breastfeeding without Baby Café."

\section{Discussion}

This report demonstrates that the Baby Café model offers community breastfeeding support that may assist mothers

Table 3. Association of MWH and SA Baby Café Attendance and Mothers Meeting/Exceeding Personal Breastfeeding Duration Goal and AAP 12-Month Duration Goal

\begin{tabular}{lccccc}
\hline & $\begin{array}{c}\text { Melrose-Wakefield } \\
\text { Baby Café }\end{array}$ & & \multicolumn{2}{c}{$\begin{array}{c}\text { SA Baby } \\
\text { Café }\end{array}$} \\
\cline { 2 - 3 } \cline { 6 - 7 } & \multicolumn{2}{c}{$n 150$} & & & $n 409$ \\
\hline Number of visits & $<5$ & $\geq 5$ & & $<5$ & $\geq 5$ \\
Met/exceeded own goal & $67 \%$ & $82 \%$ & & $78 \%$ & $92 \%$ \\
Met AAP goal 12 months & $58 \%$ & $70 \%$ & & $54 \%$ & $72 \%$ \\
\hline
\end{tabular}

Table 4. Comparison of MWH and SA Baby Café

3 Months and 6 Months Exclusive BReastfeeding Rates and CDC National Rates

\begin{tabular}{lccc}
\hline & $\begin{array}{c}\text { Melrose } \\
\text { Wakefield Baby } \\
\text { Café Rate }\end{array}$ & $\begin{array}{c}\text { SA Baby } \\
\text { Café Rate }\end{array}$ & $\begin{array}{c}\text { CDC } \\
\text { National } \\
\text { Rate }\end{array}$ \\
\hline $\begin{array}{c}\text { Exclusive at } \\
3 \text { months }\end{array}$ & $77 \%$ & $52 \%$ & $47 \%$ \\
$\begin{array}{c}\text { Exclusive at } \\
\quad 6 \text { months } \\
\text { Any breastfeeding } \\
\text { at 12 months }\end{array}$ & $71 \%$ & $47 \%$ & $25 \%$ \\
\hline
\end{tabular}

meeting AAP 3- and 6-month exclusivity and 12-month duration breastfeeding goals.

This report is the first, to our knowledge, to examine the outcomes of the Baby Café model. Follow-up surveys allow for evaluation of possible long-term implications for communities. A significant majority of the mothers (70\%) meeting the AAP breastfeeding duration goal attended the Baby Café five or more times, suggesting that a motivated and frequently attending breastfeeding mother may find guidance and solutions for issues that otherwise might result in breastfeeding cessation. Typical comments from mothers were "I wouldn't be breastfeeding without Baby Café"; "I was about to quit when I went, but they fixed it and I am still breastfeeding"; and "They found a tongue-tie that fixed the problem and I am still breastfeeding."

Baby Cafés facilitate discussions with open sharing of concerns and issues: offering experienced mothers' opportunities to assist first-time mothers and fostering community connections and friendships. Mothers offered comments reflecting their enjoyment of the social connection such as "I made so many friends," "It was very friendly and welcoming," "I learned something new every time," and "My best friends are now Café moms."

The demographics of the mothers in the two Baby Cafés used in this report differed widely from each other. Melrose mothers were $61 \%$ college educated, $83 \%$ Caucasian, $95 \%$ aged $>25$ years, $2 \%$ WIC recipients, and resided in a suburban area. The SA mothers were $79 \%$ high school educated, $79 \%$ Latino, $53 \%$ aged $>25$ years, $79 \%$ WIC recipients, and resided in a predominantly urban area. Despite these demographic differences, the Baby Café model was flexible enough to meet the needs of these different breastfeeding populations.

\section{Limitations}

Although the Baby Café survey demonstrates that the model may be an effective way to support breastfeeding mothers, there are limitations to the data collection methods. Undoubtedly, there is a degree of selection bias in the study population as the mothers who choose to attend the Café may well have been those who are more committed to breastfeeding and thus not representative of the entire universe of postpartum mothers. Furthermore, mothers successfully breastfeeding and with strong Café social connections may have been more likely to answer the survey versus mothers with possible negative feelings over their premature cessation of breastfeeding. Survey questions categorized Café 
attendance (five times or more, or less than five times) and more specific cutoffs could have been assessed if the data were continuous and not binary. Complete follow-up phone and e-mail interviews are challenging to collect, with variable response rates.

Improved Café survey collection tools are currently being developed and tested. The collection of accurate supplementation data from mothers contains inherent risk due to the memory recall of single isolated events, which occur in the hospital immediately after birth, or in crisis moments such as trauma to nipple tissue. A 2014 study found that no single indicator will provide a complete and accurate measure of how many days of exclusive breastfeeding infants have received. ${ }^{11}$ Although our data use only outcomes collected at two Baby Cafés, there is great potential for tapping the data from the growing number of (currently 15) Baby Café's survey data for future in-depth analysis.

\section{Conclusions}

The Baby Café model is suitable for a range of communities and, by offering available opportunities for free professional community-based assistance and support, may help mothers reach their stated breastfeeding goals.

\section{Ethical Statement}

This study was conducted in accordance with prevailing ethical principles and was approved by Melrose-Wakefield Hospital's (Melrose Wakefield Health care, formerly Hallmark Health System) Institutional Review Board (IRB).

\section{Disclosure Statement}

No competing financial interests exist.

\section{Funding Information}

No funding provided.

\section{References}

1. International Baby Food Network. (1981). International Code of Marketing of Breastmilk Substitutes. https://www .ibfan.org/international-code (accessed July 25, 2019).
2. Baby Friendly USA. (2012). The Ten Steps to Successful Breastfeeding. http://babyfriendlyusa.org/aboutbaby-friendly-hospital-initiative/the-ten-steps (accessed July 25, 2019).

3. Center for Disease Control and Prevention. (2018). Breastfeeding Report Card Progressing Toward National Breastfeeding Goals. www.cdc.gov/breastfeeding/data/report card.htm (accessed January 16, 2019).

4. Center for Disease Control and Prevention. (2016). Healthy People 2020 Objectives for the Nation. www.cdc .gov/breastfeeding/policy/hp2020.htm (accessed July 25, 2019).

5. U.S. Department of Health and Human Services. (2011). Executive Summary: The Surgeon General's Call To Action to Support Breastfeeding. www.surgeongeneral.gov/ library/calls/breastfeeding (accessed January 16, 2019).

6. Belay BA, Allen J, Williams N, et al. Promoting Women's Health in Hospitals: A focus on breastfeeding and lactation support for employees and patients. J Womens Health 2013;22:1-4.

7. Flagg JS, Balbier E, Blakey C. Impact of mother's breastfeeding support: Lactation support provided in a group setting. J Preg Child Health 2016;3:1-4.

8. Kauonen MH.-T. A systematic review of peer support interventions for breastfeeding. J Clin Nurs 2012;21:19431954.

9. Lee Y, Chang G, Chang H, Effects of education and support groups organized by IBCLCs in early postpartum on breastfeeding. Midwifery 2019;75:5-11.

10. American Academy of Pediatrics. Bright Futures: Guidelines for Health Supervision of Infants, Children, and Adolescents. In: Bright Futures Guidelines, 4th ed. S.J. Hagan JF, ed. Elk Grove, IL: 2017.

11. Greiner T. Exclusive breastfeeding: Measurement and indicators. Int Breastfeeding J 2014;9:18-23.

Address correspondence to: Lucia A. Jenkins, RN, IBCLC, RLC Baby Café USA, Inc. 16 Hawthorne Street Wakefield, MA 01880

USA

E-mail: lucia@babycaféusa.org 